\title{
Fatal Asymmetric Interstitial Lung Disease after Erlotinib for Lung Cancer
}

\author{
Shaohua Ren ${ }^{\mathrm{a}}$ Yuan $\mathrm{Li}^{\mathrm{a}}$ Weiwen $\mathrm{Li}^{\mathrm{a}}$ Zhongwei Zhao ${ }^{\mathrm{b}}$ Chunxian Jin ${ }^{\mathrm{b}}$ \\ Dengke Zhang ${ }^{b}$ \\ Departments of ${ }^{a}$ Respiratory Medicine and ${ }^{b}$ Radiology, Lishui Central Hospital, Lishui, PR China
}

\section{Established Facts}

- Drug-associated interstitial lung disease is a rare, but serious and often fatal adverse reaction to erlotinib, one of the oral epidermal growth factor receptor tyrosine kinase inhibitors.

- Lung injury may be perpetuated by ventilation strategies that do not limit lung volumes and airway pressures. Autopositive end-expiratory pressure is one of the risk factors for barotrauma.

\section{Novel Insights}

- We introduce a rare condition of asymmetric interstitial lung disease in a patient treated with erlotinib for lung adenocarcinoma.

- The additional affliction of the right lung was related to the dual effects of the obstructing tumor at the right main bronchus and selective bronchial artery embolization, which resulted in overexpansion, hypoventilation and hence underperfusion.

\section{Key Words}

Lung cancer - Selective bronchial artery embolization • Erlotinib $\cdot$ Interstitial lung disease

\section{Abstract \\ Pulmonary toxicity is a known complication of erlotinib, one of the epidermal growth factor receptor tyrosine kinase in- hibitors. It consists of diverse entities such as interstitial pneumonitis, bronchiolitis obliterans with organizing pneu- monia and pulmonary fibrosis. In our report, an unusual case}

of an asymmetric interstitial lung disease was described. A 68-year-old female presented with resistant cough, hemoptysis and a right lung atelectasis on chest X-ray. She underwent selective bronchial artery embolization successfully after pharmaceutical therapy failed to stop hemoptysis. Flexible bronchoscope revealed that the opening of the right main bronchus was blocked completely by a neoplasm with a distance $<2 \mathrm{~cm}$ to the carina and the sample of bronchoscopic biopsy confirmed the diagnosis of lung adenocarcinoma (CT3N2M0). Dyspnea and asymmetric interstitial lung disease in the nontumorous lung were noted on the 6th day

\section{KARGER}

Fax +4161306 1234

E-Mail karger@karger.ch

www.karger.com
(C) 2012 S. Karger AG, Basel

0025-7931/12/0845-0431\$38.00/0

Accessible online at:

www.karger.com/res
Shaohua Ren

Department of Respiratory Medicine

Lishui Central Hospital

Lishui, Zhejiang Province 323000 (PR China)

Tel.+86578 268 1222, E-Mail yj17203@yahoo.com.cn 
of erlotinib therapy (150 mg daily) which had been efficacious in its anticancer effect. Discontinuing erlotinib use and treatment with corticosteroids could not relieve her symptoms. The patient deteriorated rapidly and died of progressive respiratory failure. We explored the mechanisms of asymmetric interstitial lung disease.

Copyright ๑ 2012 S. Karger AG, Basel

\section{Introduction}

Interstitial lung disease (ILD) is a rare but fatal complication of erlotinib which was approved recently for the treatment of advanced non-small-cell lung cancer. The distribution of disease is bilateral and symmetrical in typical ILD. We present a patient who developed an unusual type of asymmetric ILD after erlotinib treatment for her lung adenocarcinoma.

\section{Case Report}

A 68-year-old female was admitted with a history of resistant cough and hemoptysis for 2 months. Physical examination revealed normal vital signs. Chest auscultation was significant for no breathing sound in the right lung, and jugular venous distention was not evident. Blood routine, biochemical tests and electrocardiogram were all within a normal range. An echocardiogram showed normal left ventricular systolic and diastolic functions. Chest X-ray (CXR) displayed a right lung atelectasis (fig. 1a). She underwent selective right bronchial artery embolization (BAE) successfully for her refractory hemoptysis as pharmaceutical therapy had failed to stop bleeding (fig. 1b, c). Flexible bronchoscope showed a neoplasm obstructing the entire opening of the right main bronchus with a distance $<2 \mathrm{~cm}$ from the carina (fig. 1d). Bronchoscopic biopsy histology led to the diagnosis of adenocarcinoma (cT3N2M0).

The patient began therapy with erlotinib, $150 \mathrm{mg}$ daily, and within 5 days her condition improved dramatically. On the 6th day, she had a good appetite and normal temperature without any signs of infection, but complained of dyspnea. Velcro rales could be heard at the bottom of the left lung. The blood routine was within a normal range. Chest CT demonstrated resolution of the atelectasis and the right lung had reexpanded well, but there was a large area of ground-glass attenuation (GGA), mainly in the left

Fig. 1. a CXR showed the right lung atelectasis at presentation. b Selective intercostobronchial trunk angiogram revealed hypertrophy in the trunk of the right bronchial artery; its branches were tortuous and of disorderly distribution. c After the injection of a gelatine sponge particle $(1,000-1,400 \mu \mathrm{m}$ in size) soaked in contrast medium, the branches of the right bronchial artery were obstructed completely. d Bronchoscopy showed a neoplasm completely blocking the opening of the right main bronchus.
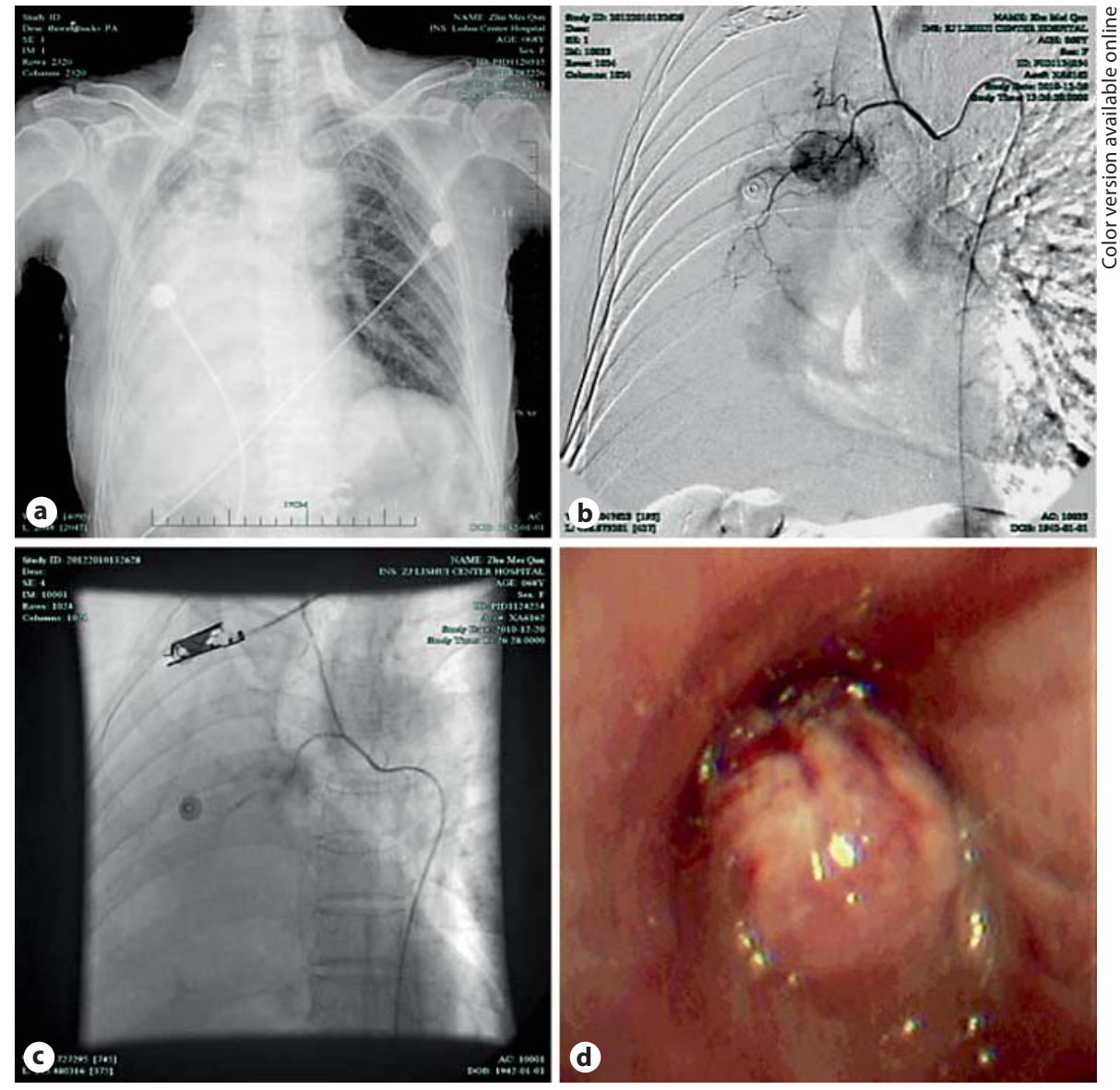
lung (fig. 2a). Erlotinib-associated ILD was considered and the drug was discontinued immediately (it had been taken for 6 days in total). The patient was started on $250 \mathrm{mg}$ of methylprednisolone administered intravenously, every $12 \mathrm{~h}$. Despite all the treatments, including corticosteroids, oxygen supply and jet ventilation, her progressive dyspnea with hypoxemia caused a rapid deterioration of her condition. The arterial blood gas under a nonrebreathing mask $\mathrm{O}_{2}$ supply $\left(\mathrm{FiO}_{2}=100 \%\right)$ was: $\mathrm{pH} 7.450$, $\mathrm{PaO}_{2} 54.5 \mathrm{~mm} \mathrm{Hg}, \mathrm{PaCO}_{2} 24.1 \mathrm{~mm} \mathrm{Hg}$ and $\mathrm{SaO}_{2} 80 \%$. Chest CT taken 4 days later revealed a diffuse distribution of GGA with a mosaic pattern and consolidation in the left lung, and GGA became more obvious at the base of the lower lobe of the right lung (fig. 2b). All of these abnormalities progressed rapidly on CXRs in the following days (fig. $2 c, d$ ). She refused mechanical ventilation, was discharged and died at home.

\section{Discussion}

The patient did not undergo chemotherapy, radiotherapy or receive any other agent that could potentially damage lung tissues. There were no signs of infection or cardiogenic pulmonary edema. Considering the temporal relationship between the use of erlotinib and the onset of symptoms, it is reasonable to conclude that the fatal ILD in the patient was induced by erlotinib $[1,2]$.

Erlotinib, an epidermal growth factor receptor tyrosine kinase inhibitor, is an effective antitumor agent for the treatment of non-small-cell lung cancer, especially in Asians. Lately, it is recommended as a first-line treatment for patients with advanced epidermal growth factor receptor mutation-positive non-small-cell lung cancer [3]. ILD is a rare but potentially life-threatening complication of erlotinib therapy with an overall incidence about $0.2-$ $6.5 \%$ in different studies $[4,5]$.

It is intriguing that the involvement of GGA was confined in the unilateral (or very asymmetric) left lung. Asymmetric ILD is not commonly seen, but does exist rarely [6-11]. Padley et al. [6] described an asymmetric form of acute respiratory distress syndrome (ARDS) following pulmonary resection. The authors found that density increased more in the nonoperated lung than in the operated lung in the patients who developed ARDS following lobectomy. This was considered as an asym-
Fig. 2. a Chest CT on the 6th day of erlotinib therapy revealed the right lung had reexpanded well and was overinflated, but there was a large area of GGA mainly in the left lung, and small cloudy shadows subpleurally at the base of the lower lobe of the right lung. $\mathbf{b}$ Chest CT taken on the 4 th day after discontinuing erlotinib revealed a diffuse distribution of GGA with a mosaic pattern and consolidation in the left lung, and the opacities at the lower lobe of the right lung were enlarged. c, d GGA was increasing gradually in the right lung while the left showed up as 'white lung' on CXRs on days 6 and 8, respectively, after discontinuing erlotinib.
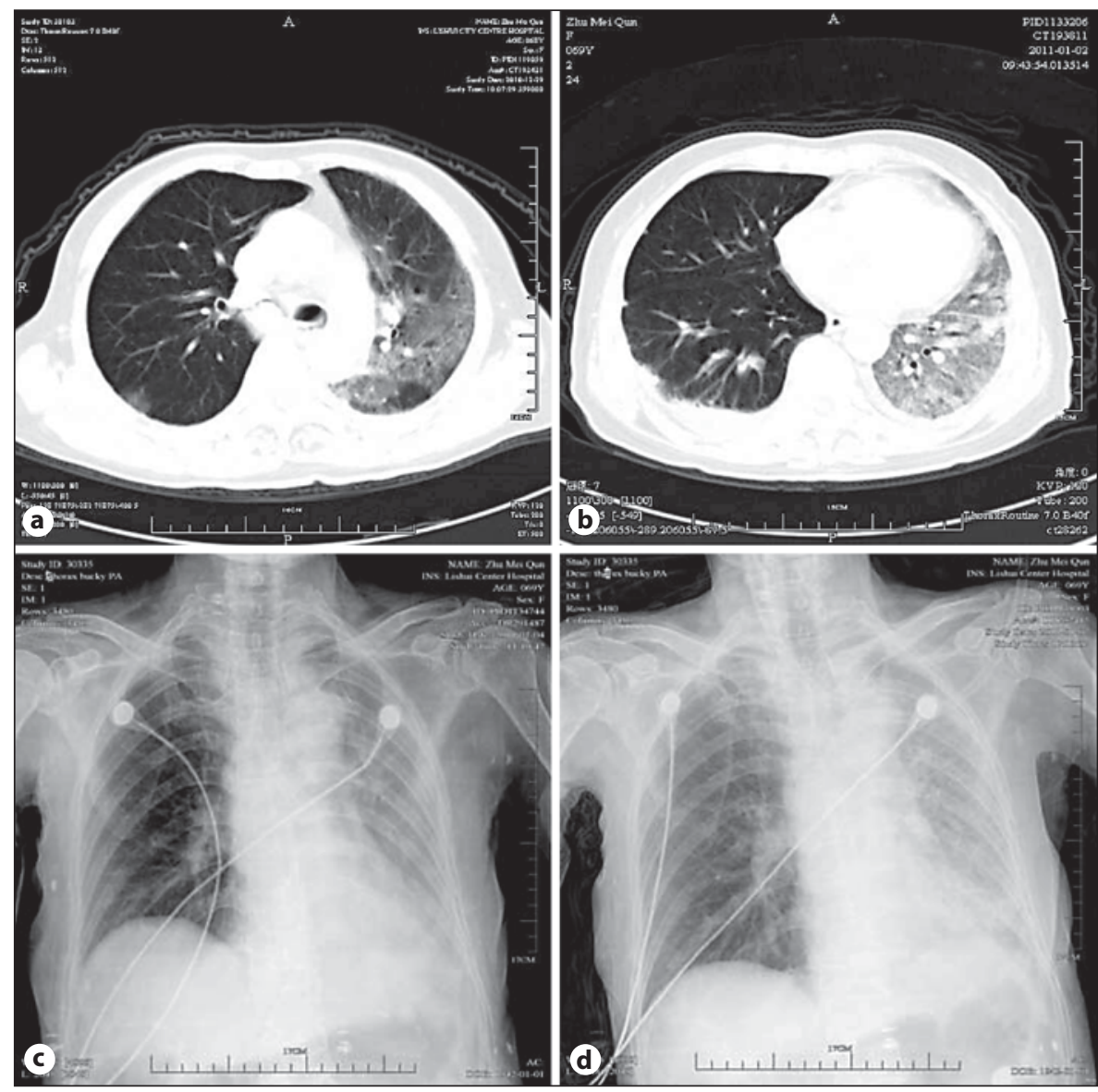
metric form of ARDS rather than compensatory hyperexpansion of the residual lung on the operated side. Kondoh et al. [7] also found that in 5/236 patients with interstitial pneumonia who underwent a surgical lung biopsy, the extent of the parenchymal involvement was significantly greater on the nonoperated side $(\mathrm{p}=0.0251)$ in all patients. The etiology of asymmetric ARDS or the extent of the parenchymal involvement was mainly related to intraoperative respiratory management, i.e. single-lung ventilation, which brought on high pressure or barotrauma and oxygen toxicity in the nonoperated lung $[6,7]$. Asymmetric ILD is also seen under nonsurgery conditions. Acid reflux and nonacid reflux in gastroesophageal reflux may proceed to the development of idiopathic pulmonary fibrosis (IPF) [8]. In a recent study, Tcherakian et al. [9] found the rates of gastroesophageal reflux and acute exacerbations were significantly higher in the patients with asymmetrical IPF than in those with symmetrical IPF. Most of the patients with asymmetrical IPF $(62.5 \%)$ showed a predominance of fibrosis in the right lung. Furthermore, the side of the fibrosis, whether right or left, matched with the patient's preferred posture for falling asleep in $94 \%$ of the asymmetrical IPF cases. Repeated microaspirations associated with gastroesophageal reflux may be locoregional factors in the development of asymmetrical IPF and acute exacerbations. Unilateral usual interstitial pneumonia and unilateral IPF were occasionally reported in the patients with slowgrowing pulmonary artery sarcoma or congenital absence of the right interlobar pulmonary artery, respectively. Chronic pulmonary ischemia associated with systemic collateral vessels was hypothesized for the pathogenesis of the diseases $[10,11]$.

As in those cases under surgery conditions, the additional affliction of the right lung in our case was due to the apparent check-valve effect of the obstructing tumor at the opening of right main bronchus which resulted in overexpansion, hypoventilation and hence underperfusion of the right lung. GGA at the bottom of the right lung was as a result of relatively higher pressure and ventilation at the base in a respiratory cycle.
Apart from the above, BAE may also play a role in the protection of the right lung by reducing the lung blood supply. Blockade of bronchial blood flow has been observed as minimizing pulmonary injury in sheep [12]. In humans, although the bronchial arteries make up only $1 \%$ of cardiac output, the function of BAE is more than providing nutrition for the walls of the bronchi, pulmonary arteries and veins. The bronchopulmonary arterial anastomoses can respond in the form of hypertrophy and perform a vital, adaptable function; more blood can be directed through the anastomoses toward the pulmonary artery to poorly ventilated or atelectatic areas in order to feed the parenchyma where its own capillaries would not carry oxygenated blood [13]. Furthermore, the diameter of the bronchial arteries might increase in the patient because of the presence of hemoptysis [14]. BAE carried out then might not only reduce the blood supply to the bronchial tumor, but also to the rest of the right lung, where a collateral circulation between the bronchial and pulmonary circulation was developed anatomically after chronic obstruction of the pulmonary arterial system by the lung tumor. However, the exact mechanism of how the reduction of lung blood supply by BAE would minimize pulmonary injury is not yet known.

In a recent study, Ter et al. [15] found that the development of the fatal ILD might be associated with high erlotinib and metabolite levels. It is possible that BAE also provides unintended protection of the right lung in our case. BAE reduced the blood supply to the bronchial tumor, joined together with the check-valve effect of the obstructing tumor, somehow decreasing the blood perfusion and lowering drug distribution in the whole right lung accordingly, and thus finally producing less toxicity in the right lung.

\section{Financial Disclosure and Conflicts of Interest}

The authors have nothing to disclose.
References
1 Camus P, Fanton A, Bonniaud Ph, et al: Interstitial lung disease induced by drugs and radiation. Respiration 2004;71:301-326.

$\checkmark 2$ Camus P, Kudoh S, Ebina M: Interstitial lung disease associated with drug therapy. $\mathrm{Br} \mathrm{J}$ Cancer 2004;91:S18-S23.

- 3 Zhou C, Wu YL, Chen G, et al: Erlotinib versus chemotherapy as first-line treatment for patients with advanced EGFR mutation-positive non-small-cell lung cancer (OPTIMAL, CTONG-0802): a multicentre, open-label, randomised, phase III study. Lancet Oncol 2011;12:735-742.

4 Peerzada MM, Spiro TP, Daw HA: Pulmonary toxicities of tyrosine kinase inhibitors. Clin Adv Hematol Oncol 2011;9:824-836. 
5 Takano T, Ohe Y, Kusumoto M, et al: Risk factors for interstitial lung disease and predictive factors for tumor response in patients with advanced non-small cell lung cancer treated with gefitinib. Lung Cancer 2004;45: 93-104.

6 Padley SP, Jordan SJ, Goldstraw P, et al: Asymmetric ARDS following pulmonary resection: CT findings - initial observations. Radiology 2002;223:468-473.

7 Kondoh Y, Taniguchi H, Kitaichi M, et al: Acute exacerbation of interstitial pneumonia following surgical lung biopsy. Respir Med 2006;100:1753-1759.

8 Pashinsky YY, Jaffin BW, Litle VR, et al: Gastroesophageal reflux disease and idiopathic pulmonary fibrosis. Mt Sinai J Med 2009;76: $24-29$. $\checkmark 9$ Tcherakian C, Cottin V, Brillet PY, et al: Progression of idiopathic pulmonary fibrosis: lessons from asymmetrical disease. Thorax 2011;66:226-231.

10 Chong S, Kim TS, Chung MP, et al: Unilateral usual interstitial pneumonia associated with sarcoma of the pulmonary artery. AJR Am J Roentgenol 2007;189:W221-W223.

11 Ryu DS, Ahn JH, Choi SJ, et al: Congenital absence of the right interlobar pulmonary artery: HRCT findings. J Thorac Imaging 2008;23:292-294.
12 Traber DL, Hawkins HK, Enkhbaatarm P, et al: The role of the bronchial circulation in the acute lung injury resulting from burn and smoke inhalation. Pulm Pharmacol Ther 2007;20:163-166.

13 Pump KK: Distribution of bronchial arteries in the human lung. Chest 1972;62:447-451.

14 Kotoulas C, Melachrinou M, Konstantinou GN, et al: Bronchial arteries: an arteriosclerosis-resistant circulation. Respiration 2010; 79:333-339.

15 Ter Heine R, van den Bosch RT, SchaeferProkop CM, et al: Fatal interstitial lung disease associated with high erlotinib and metabolite levels. A case report and a review of the literature. Lung Cancer 2012;75:391-397. 